\title{
Resultados del tratamiento con neoadyuvancia del cáncer de recto localmente avanzado*
}

\author{
Drs. GUILLERMO BANNURA C. ${ }^{1}$, ALEJANDRO BARRERA E. ${ }^{1}$, \\ CARLOS MELO L. ${ }^{1}$, JAIME CONTRERAS P. ${ }^{1}$, DANIEL SOTO C. ${ }^{1}$ \\ 1 Servicio y Departamento de Cirugía, Hospital Clínico San Borja Arriarán, Campus Centro, Facultad de Medicina, \\ Universidad de Chile. \\ Santiago, Chile.
}

\begin{abstract}
\section{Preoperative chemoradiotherapy in rectal cancer. experience in 76 patients}

Background: Preoperative chemoradiotherapy is helpful as an adjuvant treatment for locally advanced rectal carcinoma. Aim: To report the results of a prospective series of patients with rectal carcinoma treated with chemoradiotherapy and surgical excision. Material and Methods: Seventy six patients aged 28 to 84 years (51\% women), with locally advanced rectal cancer (T3-T4 or N+) subjected to curative surgery and treated with radiotherapy and 5 fluoruracil with leucovorin, were included in this series. Results: Mean hospital stay was $12.5 \pm 7.2$ days, $37 \%$ had operative complications and no patient died. The downstaging effect was more marked for local invasion than for lymph node involvement. In $21 \%$ of cases, a complete pathological response was achieved and no patient has had a relapse during a 59 months follow up. Conclusions: Among patients with rectal cancer, preoperative chemoradiotherapy does not increase surgical complications and has a marked impact in tumor downstaging.
\end{abstract}

Key words: Rectal cancer, chemotherapy, radiotherapy.

\section{Resumen}

Antecedentes: El tratamiento del cáncer de recto bajo localmente avanzado ha cambiado en la última década hacia la neoadyuvancia con radioquimioterapia (RQT) preoperatoria. Objetivo: Dar a conocer los resultados de una serie prospectiva de casos tratados con RQT preoperatoria más cirugía radical con resección total del mesorrecto. Pacientes y Método: se incluye los pacientes con un cáncer de recto medio e inferior localmente avanzado (T3-T4 y/o N+) tratados con intención curativa con radioterapia más 5-FU con leucovorina. Se detalla el impacto de la RQT en la pieza operatoria comparado con una serie similar sin radioterapia previa. Resultados: Se trata de 76 pacientes (51,3\% mujeres) con un promedio etario de 61,9 años (extremos 28-84). El tiempo de hospitalización promedio fue 12,55 días (DE: 7,19; extremos 4-35), la morbilidad global de la serie fue $37 \%$, no hubo mortalidad operatoria. El efecto del downstaging fue más marcado en el factor $\mathrm{T}$ que en el compromiso linfonodal y en el $21 \%$ de los casos se logró una respuesta patológica completa (RPC) o casi completa (RP), sin recidiva en este subgrupo luego de un seguimiento promedio de 59 meses. Conclusión: En esta serie la RQT preoperatoria no aumenta la morbilidad perioperatoria, tiene un impacto marcado en el downstaging patológico y la RPC o RP casi completa se asocia positivamente con la sobrevida a largo plazo.

Palabras clave: Cáncer de recto, neoadyuvancia, recidiva local, sobrevida.

*Recibido el 5 de Enero de 2010 y aceptado para publicación el 29 de Abril de 2010.

Correspondencia: Dr. Guillermo Bannura C.

Las Limas 1622, Santiago, Chile.

E-mail: gbannura@vtr.net 


\section{Introducción}

El enfoque del tratamiento del cáncer del recto ha sufrido cambios significativos en los últimos 20 años. De acuerdo con la Conferencia de consenso de 1990 del NIH se instituyó la radioquimioterapia (RQT) postoperatoria como el estándar terapéutico de los pacientes en estadios II y III ${ }^{1}$. Un meta-análisis efectuado en 2000 destaca que la radioterapia preoperatoria mejora la sobrevida global comparada con la cirugía exclusiva y que la RQT preoperatoria parece tener mejores resultados que la postoperatoria $^{2}$. Un estudio prospectivo multicéntrico y aleatorizado conducido en Alemania y publicado en 2004, concluye que la RQT preoperatoria reduce el riesgo de recidiva local con menos toxicidad que el tratamiento postoperatorio ${ }^{3}$. A pesar de las serias limitaciones metodológicas del estudio y el reconocimiento que esta terapia sólo mejora el control loco-regional sin beneficios en la sobrevida a largo plazo, el esquema de neoadyuvancia en el manejo del cáncer rectal localmente avanzado se ha impuesto progresivamente y actualmente es considerado el tratamiento estándar en los tumores T3-T4 y/o N $+^{4}$.

Las series presentadas en la última década en nuestro medio son muy heterogéneas. Algunas incluyen tumores del recto superior, resecciones locales y pacientes tratados con esquemas de radioterapia postoperatoria, mientras que otras se focalizan en los resultados inmediatos o los hallazgos anatomopatológicos sin una estimación de la sobrevida a largo plazo, lo que impide sacar conclusiones definitivas $^{5-8}$. En el consenso realizado por la Sociedad Chilena de Coloproctología en el 2005, se aceptó la neoadyuvancia seguido de cirugía radical como el tratamiento de elección en los pacientes con un cáncer de recto bajo localmente avanzado $(11 \mathrm{~cm} \mathrm{o}$ menos del margen anal), esquema terapéutico iniciado varios años antes por algunos grupos nacionales. El objetivo de este estudio es dar a conocer algunos resultados inmediatos y alejados de un protocolo prospectivo de RQT preoperatoria seguida de cirugía radical en tumores del recto medio e inferior en un hospital público docente.

\section{Pacientes y Método}

En el mes de junio de 1999 iniciamos un protocolo prospectivo no aleatorio de neoadyuvancia en cáncer de recto localmente avanzado. Se incluye todos los pacientes portadores de un adenocarcinoma ubicado en el recto inferior $(0-7 \mathrm{~cm}$ del margen anal) y recto medio $(7-11 \mathrm{~cm})$ cuyo borde inferior esté por debajo de $11 \mathrm{~cm}$ del margen anal medido con instrumento rígido. La etapificación clínica se efectuó mediante el examen clínico (tacto rectal) y la tomografía computada de tórax, abdomen y pelvis (TAC) incluyéndose todos los tumores T3/ $\mathrm{T} 4 \mathrm{y} / \mathrm{o} \mathrm{N}+$ que completaron el esquema de terapia neoadyuvante y fueron resecados con intención curativa (R0). El esquema de RQT preoperatoria consistió 4.500 Gy en 5 semanas ( 25 sesiones) más una sobreimpresión para alcanzar 5.040 Gy en la pelvis, asociado a quimioterapia con 5-FU $600 \mathrm{mg} /$ $\mathrm{m}^{2}$ en infusión continua durante la $1^{\circ}$ y la $5^{\circ}$ semana del tratamiento radiante. Luego de obtenida la cicatrización de las heridas y la recuperación del estado general, se indicó 6 ciclos de quimioterapia adyuvante con 5 -FU $450 \mathrm{mg} / \mathrm{m}^{2}$ más leucovorina 20 $\mathrm{mg} / \mathrm{m}^{2}$ por 5 días cada 28 días en los pacientes con estadio III. Se excluye los pacientes con tumores T1-T2, los casos de resección local, los tumores en estadio IV, los tumores ubicados en el tercio superior del recto (11-15 cm del margen anal) y los pacientes portadores de una poliposis.

La etapificación definitiva se realizó de acuerdo a la quinta edición de la clasificación AJCC conocida como TNM 1997 de la UICC ${ }^{9}$. Se define como respuesta patológica completa (RPC), la regresión completa del tumor y como RP casi completa, la presencia de nidos celulares aislados de acuerdo con la clasificación de Mandard ${ }^{10}$. Downstaging es la reducción del compromiso transmural (factor T) y/o del compromiso linfonodal (factor N) por el efecto de la RQT. La cirugía radical fue programada teóricamente 6 a 8 semanas luego de terminada la neoadyuvancia, la cual incluye una ligadura alta de los vasos mesentéricos inferiores, la resección total del mesorrecto y la preservación del plexo hipogástrico. La decisión de conservar el aparato esfinteriano en los tumores de recto bajo fue intra-operatoria. Todas las anastomosis fueron realizadas con suturas mecánicas mediante la técnica de doble grapadora. Los pacientes con una anastomosis ultrabaja (menos de $5 \mathrm{~cm}$ del margen anal) y/o con problemas técnicos de la anastomosis fueron protegidos con una ileostomía en asa. Se compara los niveles del antígeno carcinoembrionario (CEA) previo al esquema de neoadyuvancia y el control preoperatorio. Se define recidiva local como la reaparición de tumor en la pelvis y como recidiva a distancia la carcinomatosis peritoneal, las metástasis en pulmón o hígado y el compromiso de los ganglios lumbo-aórticos.

Para el análisis estadístico descriptivo se utilizó la prueba de $\mathrm{Chi}^{2}$ de Pearson y el $t$ test de Student para determinar asociaciones entre dos variables, según corresponda. Las curvas de sobrevida fueron estimadas según el método de Kaplan-Meier y para comparar las curvas de sobrevida se empleó el test $\log$-rank. Un $\mathrm{p} \leq 0,05$ se consideró estadísticamente significativo. 


\section{Resultados}

En el período de 8 años señalado se intervinieron con intención curativa en forma consecutiva 76 pacientes $(51,3 \%$ mujeres $)$ por cáncer de recto medio e inferior sometidos a RQT preoperatoria, con un promedio etario de 61,9 años (extremos 28$84)$, el $48,6 \%$ de ellos con una o más condiciones co-mórbidas, destacando la hipertensión arterial en $26 \%$, la limitación crónica del flujo aéreo (LCFA) en $3 \%$ y diabetes mellitus en $5 \%$. Tres pacientes debutan con una fístula recto-vaginal neoplásica y 1 paciente requirió de una colostomía sigmoidea por obstrucción aguda. El examen clínico y la endoscopia se complementó en el $100 \%$ de los casos con una TAC, con una ultrasonografía endorrectal (USER) en $15(20 \%)$ y una resonancia magnética (RM) en 3. El CEA pre-tratamiento promedio fue $15,1 \mathrm{ng} / \mathrm{ml}$ (desviación estándar (DE) 66,1; extremos 0,7-566) y fue superior a $5 \mathrm{ng} / \mathrm{dl}$ en el $36 \%$ de los casos. En 48 pacientes se pudo comparar el CEA previo al tratamiento y preoperatorio (post RQT). El CEA se mantuvo sin variaciones significativas en 13 pacientes de los cuales 4 fallecen con recidiva a los $43,44,51$ y 43 meses, respectivamente. El CEA previo bajó más de un $50 \%$ en 27 casos luego de la neoadyuvancia, falleciendo 1 de 6 pacientes con un CEA normal y 4 de 21 pacientes con CEA previo elevado. El CEA se elevó luego de la RQT en 8 pacientes, 4 de ellos con CEA normal ( 1 fallecido) y 4 con CEA previo > $5 \mathrm{ng} / \mathrm{ml}$ (2 fallecidos).

El intervalo entre la RQT y la cirugía radical fue en promedio de 11,5 semanas (extremos 6-25) y en 24 pacientes $(31,5 \%)$ la intervención se efectuó después de 12 semanas. Esta demora se debió por enfermedades intercurrentes, problemas administrativos o por decisión del paciente. La cirugía radical fue con conservación esfinteriana en 54 pacientes (71\%), 28 de ellos $(52 \%)$ con una ileostomía de protección (incluye 10 pacientes con exenteración pélvica posterior). La altura promedio de la anastomosis al margen anal fue $4,8 \mathrm{~cm}$ (extremos 1-8). En los 22 pacientes con una colostomía definitiva se realizó una resección abdominoperineal en 18 casos, una operación de Hartmann en 3 y una exenteración pélvica total en 1 caso. El tiempo de hospitalización promedio fue 12,55 días (DE: 7,19; extremos 4-35). La morbilidad global de la serie fue $37 \%$, motivo por el cual se reopera 4 pacientes $(5,2 \%)$, la dehiscencia anastomótica fue 5,5\% (3/54), todos con ileostomía en asa y resueltas con manejo médico conservador y no hubo mortalidad operatoria.

El impacto de la radioterapia se advierte en el estudio histopatológico de la serie comparado con una serie sin neoadyuvancia ${ }^{5}$ (Tabla 1 ). No hubo diferencias en cuanto a la edad promedio $(61,9 / 63$; $\mathrm{p}=0,5)$, porcentaje de mujeres $(51,3 / 52 ; \mathrm{p}=0,57)$, albúmina preoperatoria $(4,06 / 3,92 ; p=1,0)$ y CEA preoperatorio promedio $(15,1 / 10,82 \mathrm{ng} / \mathrm{ml} ; \mathrm{p}=0,09)$ entre ambas series. La etapificación definitiva reveló que en 10 pacientes $(13 \%)$ se logró una RPC y en 6 $(7,9 \%)$ pacientes adicionales hubo RP casi completa. La descripción macroscópica de los 10 pacientes con RPC señala 3 lesiones deprimidas (tipo IIC) (Figura 1), 6 lesiones ulceradas + depresión (IIC + III) y 1 cicatriz fibrosa de $8 \mathrm{~cm}$. El número promedio de ganglios examinados fue 8,9 (extremos $0-29$ ) y el promedio de ganglios metastásicos fue 0,84 (extremos 1-20). Veinte pacientes (26\%) tenían linfonodos

Tabla 1. Impacto de la RQT preoperatoria (downstaging)

\begin{tabular}{|c|c|c|c|c|}
\hline \multicolumn{2}{|l|}{ Variable } & $\begin{array}{l}\text { RQT preop } \\
\quad n=76\end{array}$ & $\begin{array}{c}\text { Sin RQT preop } \\
\mathbf{n}=\mathbf{8 8}\end{array}$ & p \\
\hline \multicolumn{2}{|c|}{ Tamaño tumor $(\mathrm{cm})$ promedio } & 3,31 & 6,59 & $<0,0001$ \\
\hline \multicolumn{2}{|c|}{ Tumores poco diferenciados e indiferenciados (\%) } & 5,2 & 14 & 0,04 \\
\hline \multicolumn{2}{|c|}{$\mathrm{N}^{\circ}$ ganglios examinados (promedio) } & 8,89 & 16,7 & $<0,0001$ \\
\hline \multicolumn{2}{|c|}{$\%$ ganglios positivos } & 26 & 48 & 0,001 \\
\hline $\mathrm{n}$ & $\begin{array}{l}\text { T0 } \\
\text { T1 } \\
\text { T2 } \\
\text { T3 } \\
\text { T4 }\end{array}$ & $\begin{array}{r}10 \\
6 \\
13 \\
41 \\
6\end{array}$ & $\begin{array}{r}0 \\
0 \\
4 \\
69 \\
15\end{array}$ & $<0,0001$ \\
\hline $\mathrm{n} \quad(\%)$ & $\begin{array}{l}0 \\
\text { I } \\
\text { II } \\
\text { III }\end{array}$ & $\begin{array}{ll}10 & (13) \\
15 & (20) \\
31 & (41) \\
20 & (26)\end{array}$ & $\begin{array}{r}0 \\
6(7) \\
40(45) \\
42(48)\end{array}$ & $<0,0001$ \\
\hline
\end{tabular}




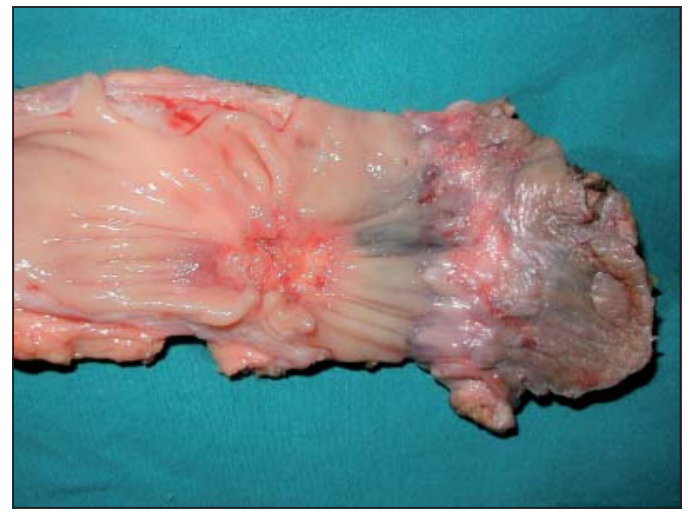

Figura 1. Lesión residual tipo II C con neoadyuvancia: RPC.

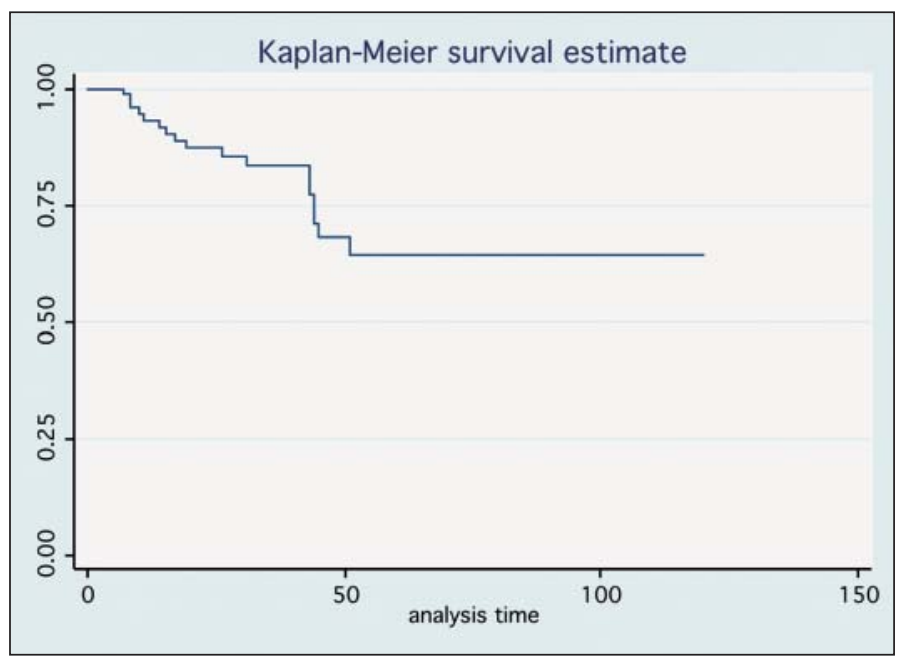

Figura 2. Sobrevida global según Kaplan-Meier.

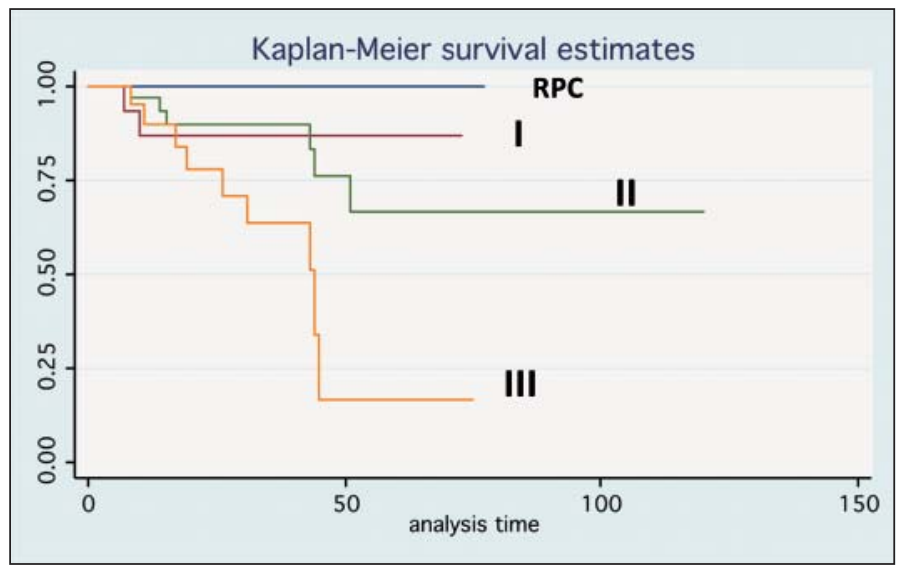

Figura 3. Sobrevida estimada según TNM. metastásicos (15 con 1 a 3 ganglios positivos (N1) y 5 con 4 o más ganglios afectados (N2). El tamaño promedio de la lesión fue 3,31 cm (DE: 1,87; extremos 0,6-10).

El seguimiento promedio de la serie fue 39 meses (DE: 24,3; extremos 7-120). Veintitrés pacientes (20 estadio III y 3 estadio II) completaron 6 ciclos de quimioterapia postoperatoria con 5 $\mathrm{FU}+$ Leucovorina. En dicho período de seguimiento se pesquisa una RL exclusiva en 3 pacientes y RL + RD en 4 casos, con una RL global de 7 casos $(9 \%)$. La RD exclusiva fue de 11 pacientes, lo que da una recidiva global a distancia de 15 casos $(20 \%)$. De este modo, la recidiva global de la serie (RL + RD) fue 23,7\% (18 casos), falleciendo por cáncer 17 pacientes (22,3\%). Hubo 1 paciente sometido a cirugía radical de rescate por una RL exclusiva con sobrevida de 70 meses y actualmente sin evidencia de enfermedad. No hubo recidiva en los pacientes con RPC o RP casi completa (21\% de la serie), subgrupo con un seguimiento promedio de 59 meses (extremos 1174). La sobrevida actuarial a 5 años para la serie global fue $65 \%$, cifra que alcanza al $100 \%$ para los pacientes con RPC, 90\% para los estadios I, 66\% para los estadios II y $20 \%$ para los estadios III $(p=0,005)$. Las curvas de supervivencia global y de acuerdo al TNM se observa en las Figuras 2 y 3.

\section{Discusión}

La neoadyuvancia se ha convertido en el tratamiento estándar para los tumores de recto medio e inferior localmente avanzados en la última década. Las ventajas teóricas del tratamiento preoperatorio se confirmaron en cuanto a la recidiva local en el estudio alemán de 2004 que ha fundamentado este enfoque $^{3}$, aunque no pudo demostrar la superioridad de este esquema en la supervivencia a largo plazo respecto de la RQT postoperatoria. La crítica en los aspectos metodológicos de este estudio puede resumirse en 5 puntos: 1) Incluye un $18 \%$ de pacientes con tumores del tercio superior del recto (hasta 
$16 \mathrm{~cm})$; 2) Compara dos series que difícilmente son comparables: una serie de RQT preoperatoria con una etapificación clínica (incierta), con una etapificación patológica apoyada en el estudio de una pieza irradiada (definitiva) (de hecho, el 18\% de los pacientes en el grupo de RQT postoperatoria correspondían al estadio I); 3) El 40\% de los pacientes en el grupo de RQT postoperatoria no recibió radioterapia (en parte por la razón anterior); 4) El mejor control local atribuido a la neoadyuvancia $(6 \% / 13 \%$ recidiva local) podría ser explicado entre otros factores por la calidad de la cirugía; 5) El estudio multicéntrico analizado sugiere lo anterior, si tenemos en cuenta que participaron 26 centros europeos con más de 130 cirujanos en un período de 48 meses, lo que implica un reducido número de pacientes por cirujano o, al menos, diferencias marcadas en la experticia de los participantes. A raíz de estudios suecos con neoadyuvancia ${ }^{11,12}$ y los mediocres resultados obtenidos con nuestros pacientes con el esquema de RQT postoperatoria ${ }^{5}$, iniciamos en 1999 este estudio prospectivo cuyos resultados confirman algunos aspectos interesantes de esta modalidad de manejo del cáncer de recto localmente avanzado.

Definitivamente la neoadyuvancia no aumenta en forma significativa la morbilidad postoperatoria, la cual fluctúa entre 30 y $45 \%$, con una mortalidad peri-operatoria entre 0 y $2 \%{ }^{7,8,13,14}$. Se ha sugerido que la morbilidad podría aumentar en los pacientes con una mayor regresión tumoral, lo que requiere confirmación ${ }^{15}$. El intervalo ideal para efectuar la cirugía radical es un tema controversial. Un intervalo entre 8 a 12 semanas parece ser óptimo para evaluar el impacto de la RQT en el tumor, sin un aumento de la morbilidad quirúrgica. Sin embargo, prolonga el tiempo de incapacidad del paciente y no está claro si ello tiene impacto en una mejor sobrevida ${ }^{16}$. El porcentaje de cirugía con conservación esfinteriana (71\%) de la serie es similar a otros estudios que sólo incluyen tumores de recto medio e inferior ${ }^{7,13-15}$. El comportamiento del CEA luego de la neoadyuvancia fue tan errático que las variaciones de este marcador con la RQT no orientan en cuanto al pronóstico. De cualquier manera, parece útil considerar el CEA preoperatorio en la toma de decisiones cuando está muy elevado ${ }^{17}$.

El impacto de la RQT de curso largo en la pieza operatoria es innegable (Tabla 1), destacando la disminución del tamaño tumoral, la menor obtención de linfonodos y la reducción en el porcentaje de ganglios metastásicos, lo que determina una disminución del porcentaje de pacientes en estadíos III. El significado en términos del pronóstico de este downstaging es controversial. La neoadyuvancia con RQT logra un porcentaje de pacientes con RPC o RP casi completa ( $21 \%$ en nuestra serie) que no es despreciable, lo que al parecer se correlaciona con la sobrevida ${ }^{18-20}$. Al igual que en otras series, los pacientes con esta respuesta a la radioterapia más una quimioterapia de sensibilización tienen un riesgo muy bajo de recidiva local y exhiben una sobrevida cercana al $100 \%{ }^{21}$. A ello hay que agregar que los estudios recientes sugieren que la sensibilización podría efectuarse con capecitabina oral con un beneficio terapéutico similar al tratamiento clásico con las ventajas de la administración oral ${ }^{22}$.

En la actualidad el desafío mayor es identificar los factores que permitan predecir la respuesta a la RQT preoperatoria (pacientes "radiosensibles"), lo que aún está en discusión. En un estudio retrospectivo de 562 pacientes de una Institución ${ }^{23}$, el margen circunferencial $<60 \%$ del tumor, el CEA preoperatorio $<2,5 \mathrm{ng} / \mathrm{ml}$ y la distancia del tumor al margen anal $<5 \mathrm{~cm}$ fueron considerados variables predictivas de una mejor respuesta patológica a la RQT en el análisis multivariado, aunque no se señala el impacto de esto en la supervivencia a largo plazo. Otros autores ${ }^{18-21,24}$ son enfáticos en que la RQT preoperatoria logra un downstaging patológico que impacta tanto en la recidiva local como en la sobrevida alejada. En nuestra serie y la de otros ${ }^{20,25}$ el impacto en la sobrevida del compromiso metastásico de los linfonodos queda de manifiesto en la inflexión negativa de la curva en los estadios III, lo que es estadísticamente significativo.

Entre las críticas a la neoadyuvancia, se ha señalado el riesgo de realizar un tratamiento radiante en pacientes con tumores menos avanzados, lo que es real mientras no se cuente con imágenes más precisas. En la experiencia de algunos autores, el 26\% de los tumores uT3 en la etapificación preoperatoria tenían un estadio I en el grupo sin neoadyuvancia ${ }^{19}$. En un meta-análisis del 2004 se destaca que la sensibilidad para la etapificación del factor T (compromiso transmural) de la RM y la USER alcanza al $94 \%$, con una especificidad de $86 \%$ para el USER y $69 \%$ para la RM. Sin embargo, estos exámenes no son confiables para evaluar el factor ganglionar con una sensibilidad y una especificidad que no supera el $67 \%$ y $77 \%$, respectivamente ${ }^{26}$.

En conclusión, se presenta una serie prospectiva de pacientes portadores de un cáncer localmente avanzado de recto medio e inferior sometidos a RQT previa a cirugía radical con resección total del mesorrecto con intención curativa. La intervención fue realizada luego de un intervalo promedio de 11,5 semanas sin un aumento de la morbilidad perioperatoria, con conservación del aparato esfinteriano en el $71 \%$ de los casos y sin mortalidad. El efecto del downstaging fue más marcado en el factor T que en el factor $\mathrm{N}$ (compromiso linfonodal) y en el $21 \%$ 
de los casos se logró una RPC o RP casi completa. A pesar de las limitaciones de este estudio y dadas las dificultades en cumplir cabalmente con los plazos teóricos, en esta serie la respuesta tumoral a la neoadyuvancia se asocia positivamente con la sobrevida a largo plazo y no hubo recidiva ni fallecidos en pacientes con RPC o RP casi completa, con un período de seguimiento promedio de 59 meses. La sobrevida actuarial a 5 años fue 100, 90, 66 y 20\% para los pacientes con RPC, estadios I, estadios II y estadios III, respectivamente $(p=0,005)$. En concordancia con otros autores ${ }^{25}$, el factor linfonodal en pacientes tratados con intención curativa es el factor pronóstico de mayor relevancia en el cáncer de recto localmente avanzado luego de neoadyuvancia con RQT.

\section{Referencias}

1. NIH consensus conference: Adjuvant therapy for patients with colon and rectal cancer. JAMA 1990; 264: 1440-1450.

2. Camma C, Giunta M , Fiorica M, Pagliaro L, Craxi A, Cottone M. Preoperative radiotherapy for resectable rectal cancer. A meta-analysis. JAMA 2000; 284: 10081015.

3. Sauer R, Becker H, Hohenberger W, Rödel C, Wittekind $\mathrm{C}$, Fietkau R, et al. Preoperative versus postoperative chemoradiation for rectal cancer. N Engl J Med 2004; 351: 1731-1740.

4. Ceelen W, Fierens K, Van Nieuwenhove Y, Pattyn P. Preoperative chemoradiation versus radiation alone for stage II and III resectable rectal cancer: Systematic review and meta-analysis. Int J Cancer 2009; 124: 29662972.

5. Bannura G, Contreras J, Cumsille MA, Melo C, Valencia C. Resultados del tratamiento quirúrgico del cáncer de recto: análisis de 180 pacientes. Rev Chil Cir 1998; 50: 646-654.

6. Suárez J, González P, Giannini O, Rey G, González R, Adelsdorfer C, y cols. Buen control y sobrevida post resección curativa de cáncer de recto medio e inferior. Protocolo terapéutico multimodal, selectivo. Rev Chil Cir 2003; 55: 584-590.

7. Zúñiga A, Escalona A, López-K F, Rahmer A, Álvarez M, Besa P, y cols. Cáncer de recto localmente avanzado: impacto de un protocolo de radioquimioterapia preoperatorio en los resultados quirúrgicos inmediatos. Rev Chil Cir 2001; 53: 551-556.

8. Escalona A, Zúñiga A, López-K F, Rahmer A. Radioquimioterapia preoperatoria y resección radical del cáncer localmente avanzado del recto: análisis de los hallazgos histopatológicos y su repercusión clínica. Rev Chil Cir 2003; 55: 55-59.

9. Fleming ID, Cooper JS, Henson DE, Hutter RVP, Kennedy BJ, Murphy GP, et al. Editors. American Joint
Committee on Cancer. Cancer manual staging. $5^{\text {th }}$ edition, 1997.

10. Mandard AM, Dalibard F, Mandard JC, Marnay J, Henry-Amar M, Petiot JF, et al. Pathologic assessment of tumor regression after preoperative chemoradiotherapy of esophageal carcinoma. Cancer 1994; 73: 2680-2686.

11. Martling A, Holm T, Johansson H, Cedermark B. Stockholm Colorectal Cancer Study Group. The Stockholm II trial on preoperative radiotherapy in rectal carcinoma. Cancer 2001; 92: 896-902.

12. Swedish Rectal Cancer Trial: Improved survival with preoperative radiotherapy in resectable rectal cancer. N Engl J Med 1997; 336: 980-987.

13. Alves A, Panis Y, Mathieu P, Kwiatkowski F, Slim K, Mantion G. Mortality and morbidity after surgery of mid and low rectal cancer. Gastroenterol Clin Biol 2005; 29: 509-514.

14. Valero G, Luján JA, Hernández Q, De Las Heras M, Pellicer E, Serrano A, et al. Neoadjuvant radiation and chemotherapy in rectal cancer does not increase postoperative complications. Int J Colorectal Dis 2003; 18: 495-499.

15. Horisberger K, Hofheinz RD, Palma P, Volkert AK, Rothenhoefer S, Wenz F, et al. Tumor response to neoadjuvant chemoradiation in rectal cancer: predictor for surgical morbidity? Int J Colorectal Dis 2008; 23: 257-264.

16. Veenhof AAFA, Kropman RHJ, Engel AF, Craanen ME, Meijer S, van der Peet DL, et al. Preoperative radiation therapy for locally advanced rectal cancer: a comparison between two different time intervals to surgery. Int $\mathrm{J}$ Colorectal Disease 2007; 22: 507-513.

17. Bannura G, Cumsille MA, Barrera A, Contreras J, Melo C, Soto D, y col. CEA preoperatorio como factor pronóstico en cáncer de colon y recto: análisis de 532 pacientes. Rev Chil Cir 2008; 60: 320-325.

18. Klautke G, Fietkau R. Intensified neoadjuvant radiochemotherapy for locally advanced rectal cancer: a review. Int J Colorectal Dis 2007; 22: 457-465.

19. Rosenberg R, Nekarda H, Zimmermann F, Becker K, Lordick F, Hofler H, et al. Histopathological response after preoperative radiochemotherapy in rectal carcinoma is associated with improved overall survival. J Surg Oncol 2008; 97: 8-13.

20. Quah H-M, Chou JF, Gonen M, Shia J, Schrag D, Saltz LB, et al. Pathological stage is most prognostic of disease-free survival in locally advanced rectal cancer patients after preoperative chemoradiation. Cancer 2008; 113: 57-64.

21. Suárez J, Vera R, Balén E, Gómez M, Arias F, Lera JM, et al. Pathologic response assesed by Mandard grade is a better prognostic factor than downstaging for diseasefree survival after preoperative radiochemotherapy for advanced rectal cancer. Colorectal Dis 2007; 10: 563 568 .

22. de Bruin AFJ, Nuyttens JJ, Ferenschild FTJ, Planting 
AST, Verhoef C, de Wilt JHW. Preoperative chemoradiation with capecitabine in locally advanced rectal cancer. Neth J Med 2008; 66: 71-76.

23. Das P, Skibber JM, Rodríguez-Bigas MA, Feig BW, Chang GJ, Wolff RA, et al. Predictors of tumor response and downstaging in patients who receive preoperative chemoradiation for rectal cancer. Cancer 2007; 109: 1750-1755.

24. Kim NK, Baik SH, Seong JS, Kim H, Roh JK, Lee $\mathrm{KY}$, et al. Oncologic outcomes after chemoradiation followed curative resection with tumor-specific meso- rectal excision for fixed locally advanced rectal cancer. Ann Surg 2006; 244: 1024-1030.

25. Kim NK, Kim Y-W, Min B-S, Lee K-Y, Sohn S-K, Cho C-H. Factors associated with local recurrence after neoadjuvant chemoradiation with total mesorectal excision for rectal cancer. World J Surg 2009; 33: 1741-1749.

26. Bipat S, Glas AS, Slors FJ, Zwinderman AH, Bossuyt PM, Stoker J. Rectal cancer: local staging and assessment of lymph node involvement with endoluminal US, CT, and MR imaging- a meta-analysis. Radiology 2004; 232: 773-783. 